\title{
An analysis of the Shapley Value and its Uncertainty for the Voting Game
}

\author{
Shaheen S. Fatima ${ }^{1} \quad$ Michael Wooldridge $^{1} \quad$ Nicholas R. Jennings $^{2}$ \\ ${ }^{1}$ Department of Computer Science, \\ University of Liverpool, Liverpool L69 7ZF, U.K. \\ \{S.S.Fatima, M.J.Wooldridge\}@Csc.liv.ac.uk \\ ${ }^{2}$ School of Electronics and Computer Science, \\ University of Southampton, Southampton SO17 1BJ, U.K. \\ nrj@ecs.soton.ac.uk
}

\begin{abstract}
The Shapley value provides a unique solution to coalition games and is used to evaluate a player's prospects of playing a game. Although it provides a unique solution, there is an element of uncertainty associated with this value. This uncertainty in the solution of a game provides an additional dimension for evaluating a player's prospects of playing the game. Thus, players want to know not only their Shapley value for a game, but also the associated uncertainty. Given this, our objective is to determine the Shapley value and its uncertainty and study the relationship between them for the voting game. But since the problem of determining the Shapley value for this game is \#P-complete, we first present a new polynomial time randomized method for determining the approximate Shapley value. Using this method, we compute the Shapley value and correlate it with its uncertainty so as to allow agents to compare games on the basis of both their Shapley values and the associated uncertainties. Our study shows that, a player's uncertainty first increases with its Shapley value and then decreases. This implies that the uncertainty is at its minimum when the value is at its maximum, and that agents do not always have to compromise value in order to reduce uncertainty.
\end{abstract}

\section{Introduction}

Coalition formation is the process of joining together of two or more agents so as to achieve goals that individuals on their own cannot, or to achieve them more efficiently [9]. Often, in such situations, there is more than one possible coalition and a player's payoff depends on the coalition it joins. Given this, a key problem in this area is to ensure that none of the parties in a coalition has any incentive to break away from it and join another coalition (i.e., the coalitions are stable). However, in many cases there may be more than one solution (i.e., a stable coalition). In such cases, it becomes difficult to select a single solution from among the possible ones, especially if the parties are self-interested (i.e., they have different preferences over stable coalitions).

In this context, cooperative game theory deals with the problem of coalition formation and offers a number of solution concepts that possess desirable properties like stability, fair division of joint gains, and uniqueness $[3,7]$. Multiagent systems research has used and extended these game-theoretic solutions to facilitate automated coalition 
formation $[9,14,12]$. In this work, one of the most extensively studied solution concepts is the Shapley value [13]. The Shapley value provides a unique solution and is therefore used to evaluate a player's prospects of playing a game.

Although the Shapley value provides a unique solution, it has two key drawbacks. First, for the weighted voting game that we consider, the problem of determining the Shapley value is \#P-complete [1]. Second, it provides the solution only with a limited degree of certainty [11]. Thus the uncertainty in the Shapley value provides an additional dimension for evaluating a player's prospects of playing a game and a measure of uncertainty would serve as a useful tool to investigate this aspect of a game. Characterizing a game by both its value and uncertainty is like characterising a weapon by its power and precision, or a financial stock by its expected return and risk [4].

The concept of uncertainty in the outcome of a game is not entirely new. For instance, Roth showed that the Shapley value of a game equals its utility, if and only if the underlying player preferences are neutral to both ordinary ${ }^{1}$ and strategic risk [10, 11]. Otherwise, the Shapley value is not the same as utility and is therefore insufficient for decision-making purposes. Kargin extended this concept further by introducing a measure for determining the strategic risk [4]. This measure is called the uncertainty of the Shapley value and it provides a yardstick for quantifying the strategic risk. Thus, in order for a player to make more informed decisions, it is important for it to not only know its Shapley value, but also the relation between this value and its uncertainty. However, to date, there has been no analysis of this relationship.

Given this, our objective is to analyse the relation between the Shapley value and its uncertainty for the voting game (since it is an important mechanism for multiple agents to reach consensus). However, uncertainty is defined in terms of the Shapley value (i.e., in order to find uncertainty, the Shapley value needs to be determined first). But, as we pointed out, the problem of determining the Shapley value has been shown to be \#P-complete [1]. We therefore present a new randomised method (that has polynomial time complexity) for computing the approximate Shapley value. Using this method, we determine the Shapley value and correlate it with its uncertainty. Our study shows that each player's uncertainty first increases with its Shapley value and then decreases. This implies that the uncertainty is at its minimum when the value is at its maximum, and that agents do not always have to compromise value in order to reduce uncertainty.

To our knowledge, the only work that addresses the problem of uncertainty in the Shapley value is $[10,11,4]$. While $[10,11]$ introduces the concept of strategic risk in the context of the Shapley value, [4] defines a measure (called uncertainty) for this risk. Our paper therefore makes a twofold contribution. First, we present a polynomial time method along the lines of Monte Carlo simulation (see Section 3 for details) for computing the Shapley value for the voting game. Second, using this method we compute the Shapley value and analyse its relation with uncertainty.

Section 2 defines the Shapley value and its uncertainty. Section 3 describes the weighted voting game. Section 4 to Section 7 determine the relation between the Shapley value and its uncertainty. Section 8 concludes.

\footnotetext{
${ }^{1}$ Ordinary risk involves the uncertainty that arises from the chance mechanism involved in lotteries. On the other hand, strategic risk involves the uncertainty that arises as a result of interaction in a game of strategic players (i.e., those players that are not dummy).
} 


\section{The Shapley value and its uncertainty}

We begin by introducing coalition games and then define the weighted voting game. Coalition games are of two types ([7]): those with transferable payoff and those with non-transferable payoff. A coalition game with transferable payoff, $\langle N, v\rangle$, consists of a finite set $(N=\{1,2, \ldots, n\})$ of players and a function $(v)$ that associates with every non-empty subset $S$ of $N$ (i.e., a coalition) a real number $v(S)$ (the worth of $S$ ).

For each coalition $S$, the number $v(S)$ is the total payoff that is available for division among the members of $S$ (i.e., the set of joint actions that coalition $S$ can take consists of all possible divisions of $v(S)$ among the members of $S$ ). Coalition games with nontransferable payoffs differ from ones with transferable payoffs in the following way. For the former, each coalition is associated with a set of payoff vectors that is not necessarily the set of all possible divisions of some fixed amount. In this paper, we focus on the Shapley value for a game with transferable payoffs.

Let $\mathcal{S}$ denote the set $N-\{i\}$ and $f_{i}: \mathcal{S} \rightarrow 2^{N-\{i\}}$ be a random variable that takes its values in the set of all subsets of $N-\{i\}$, and has the probability distribution function $(g)$ defined as:

$$
g\left\{f_{i}(\mathcal{S})=S\right\}=\frac{|S| !(n-|S|-1) !}{n !}
$$

The random variable $f_{i}$ is interpreted as the random choice of a coalition that player $i$ joins. A player's Shapley value [13] is defined in terms of its marginal contribution. The marginal contribution of player $i$ to coalition $S$ with $i \notin S$ is a function $\Delta_{i} v$ that acts in the following way:

$$
\Delta_{i} v(S)=v(S \cup\{i\})-v(S)
$$

Definition 1. The Shapley value $\left(\varphi_{i}\right)$ of the game $\langle N, v\rangle$ for player $i$ is the expectation (E) of its marginal contribution to a coalition that is chosen randomly, i.e., $\varphi_{i}(N, v)=$ $E\left\{\Delta_{i} v \circ f_{i}\right\}$

The Shapley value is interpreted as follows. Suppose that all the players are arranged in some order, all orderings being equally likely. Then $\varphi_{i}(N, v)$ is the expected marginal contribution, over all orderings, of player $i$ to the set of players who precede him. The uncertainty of the Shapley value, is defined as follows [4]:

Definition 2. The uncertainty $\left(\beta_{i}\right)$ for player $i$ is the variance (Var) of its marginal contribution. Thus $\beta_{i}(N, v)=\operatorname{Var}\left\{\Delta_{i} v \circ f_{i}\right\}$

Thus, while a player's Shapley value is the expectation (i.e., the mean), its uncertainty is the variance (i.e., the square of the standard deviation) of its marginal contribution. In other words, the uncertainty is the expectation of the squared difference between the actual and expected marginal contributions.

The utility of a player that is not neutral to strategic risk depends on both its Shapley value and the associated uncertainty. Furthermore, such a player's utility function is subjective and different players may have different functions for the same game. But for a given game, the relation between the Shapley value and its uncertainty is not subjective to player preferences and is the same for all players. We therefore analyse this relation for the voting game described in Section 3. 


\section{The weighted voting game}

We adopt the definition of voting game given in [7]. There is a set of $n$ players that may, for example, represent shareholders in a company or members in a parliament. The weighted voting game is a game $G=\langle N, v\rangle$ in which

$$
v(S)=\left\{\begin{array}{l}
1 \text { if } w(S) \geq q \\
0 \text { otherwise }
\end{array}\right.
$$

for some $q \in \mathbb{R}_{+}$and $w_{i} \in \mathbb{R}_{+}^{N}$, where $w(S)=\sum_{i \in S} w_{i}$ for any coalition $S$. Thus $w_{i}$ is the number of votes that player $i$ has and $q$ is the number of votes needed to win the game (i.e., the quota). For this game (denoted $\left\langle q ; w_{1}, \ldots, w_{n}\right\rangle$ ), a player's marginal contribution is either zero or one.

The problem of determining the Shapley value for the weighted voting game is \#Pcomplete [1]. A problem is \#P-hard if solving that problem is as hard as counting satisfying assignments of propositional logic formulae [8, p442]. Since \#P-completeness thus subsumes NP-completeness, this implies that computing the Shapley value for the weighted voting game will be intractable in general. To overcome this problem, two methods have been proposed: Monte Carlo simulation [5] and the method of generating functions [6]. The former method treats the number of swings $^{2}$ for each player as a random variable over a given distribution and defines the Shapley value in terms of these random variables. While this method gives the approximate Shapley value, the generating functions method is an exact procedure. Although it is an exact procedure, it requires very large arrays (i.e., it requires substantial storage space) and can only be applied to games with integer weights and quotas.

The method we present is similar to that of [5] in the sense that it is an approximation method. But the difference is that while [5] defines the Shapley value by treating a player's number of swings as a random variable, we treat the players' weights as random variables. Since the voting game is defined in terms of the players' weights and the number of swings are obtained from these weights, our method corresponds more closely to the definition of the voting game. Furthermore, it does not require large arrays and is therefore economical in terms of storage space. The proposed method has polynomial time complexity. We first consider a simple voting game in which all players have equal weight. We then extend our analysis to a game with two types of players: large and small, and finally generalise it to more than two player types.

\section{All players have equal weight}

Consider the game $\langle q ; j, \ldots, j\rangle$ with $m$ parties. Each party has $j$ seats. If $q \leq j$, then there would be no need for players to form a coalition. On the other hand, if $q=m j$ ( $m=|N|$ is the number of players), only the grand coalition is possible. Thus, the quota $(q)$ satisfies the constraint: $(j+1) \leq q \leq j(m-1)$. A majority is decisive. The value of a coalition is one if the weight of the coalition is greater than or equal to $q$, otherwise it is zero.

\footnotetext{
${ }^{2}$ A swing for a player $i$ is a pair of coalitions $(x, x \cup i)$ such that $x$ is losing but $x \cup i$ is winning.
} 


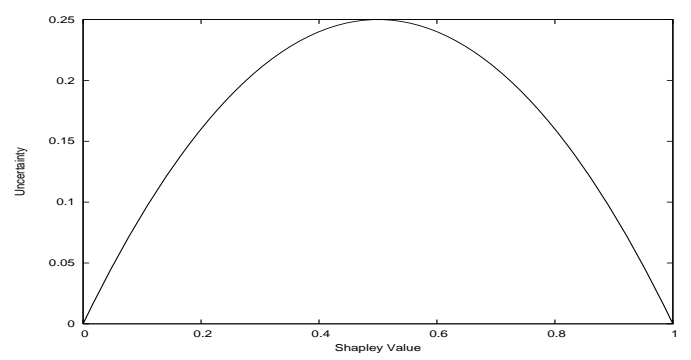

Fig. 1. Shapley value vs. uncertainty

Let $\varphi$ denote the Shapley value for a player and $\beta$ denote its uncertainty. Consider any one player. This player can join a coalition as the $i$ th member where $1 \leq i \leq m$. However, the marginal contribution of the player is 1 only if it joins a coalition as the $\lceil q / j\rceil$ th member. In all other cases, its marginal contribution is zero. Thus, the Shapley value for each player is $\varphi=1 / m$. We know from Definition 2, that a player's uncertainty is the variance of its marginal contribution. Hence, for each player, the uncertainty $(\operatorname{denoted} \beta)$ is:

$$
\beta=(1-\varphi) \varphi^{2}+(1-\varphi)^{2} \varphi
$$

Having expressed a player's uncertainty in terms of its Shapley value, we can now correlate them. To this end, Figure 1 shows how the uncertainty varies with the Shapley value. Since the Shapley value lies in the interval $[0,1]$, Figure 1 plots uncertainty for this interval. As seen in the figure, uncertainty first increases as the Shapley value increases and then decreases. Uncertainty is maximum at $\varphi=1 / 2$. The following sections analyse the voting game for the case where all parties do not have equal weight.

\section{A single large party}

Consider a parliament in which there is one party with $j>1$ seats, and $m$ parties each with one seat. Thus, there are two types of players: large (with weight $j$ ) and small (with unit weight). The quota for this game is $q$, i.e., we have a game of the form $\langle q ; j, 1,1, \ldots, 1\rangle$. The total number of players is $(m+1)$. The value of a coalition is one if the weight of the coalition is greater than or equal to $q$, otherwise it is zero. Let $\varphi_{l}$ denote the Shapley value for the large player and $\varphi_{s}$ that for each small player. As we will show, the Shapley value of this game depends on whether or not $q$ is greater than $m$. We therefore study the two possible cases: $q \leq m$ and $q>m$ :

1. Consider $q \leq m$ first. The smallest possible value for $q$ is $j+1$. This is because, if $q \leq j$, then the large party can win the election on its own, without the need for a coalition. Thus, the quota for the game satisfies the relation $j+1 \leq q \leq m+j-1$. Also, the lower and upper limits for $j$ are 2 and $(q-1)$ respectively. The lower limit is 2 because the weight of the large party has to be greater than each small 
one. Furthermore, the weight of the large party cannot be greater than $q$, since in that case there would be no need for the large party to form a coalition. Recall that for our voting game, a player's marginal contribution to a coalition has only two possible values: zero or one.

Consider the large party. This party can join a coalition as the $i$ th member where $i$ satisfies $1 \leq i \leq(m+1)$. However, the marginal contribution of the large party is one if it joins a coalition as the $i$ th member where $i$ satisfies the condition $(q-j+1) \leq i \leq q$. In all the remaining cases, its marginal contribution is zero. Thus, out of the total $(m+1)$ possible cases, its marginal contribution is one in $j$ cases. Hence, the Shapley value of the large party is:

$$
\varphi_{l}=j /(m+1)
$$

Consider a small player. For a small player, the marginal contribution is one in two cases. First, if it joins a coalition (that already has the large party in it) as the $(q-j+1)$ th member. Out of the $(m+1)$ ! possible permutations, the number of permutations that satisfy this condition is $(q-j)(m-1)$ !. Second, if it joins a coalition (consisting of $q-1$ small players) as the $q$ th member. The number of permutations that satisfy this condition is $(m-q+1)(m-1)$ !. Hence, the Shapley value of each small party is:

$$
\varphi_{s}=(m-j+1) / m(m+1)
$$

Using Definition 2, we get the uncertainty for the large party as:

$$
\beta_{l}=\left(1-\varphi_{l}\right) \varphi_{l}^{2}+\varphi_{l}\left(1-\varphi_{l}\right)^{2}
$$

For each small party, the uncertainty is:

$$
\beta_{s}=\left(1-\varphi_{s}\right) \varphi_{s}^{2}+\varphi_{s}\left(1-\varphi_{s}\right)^{2}
$$

2. Consider $q>m$. As before, the quota satisfies the relation $j+1 \leq q \leq m+j-1$. Also, $2 \leq j \leq(q-1)$. Consider the large party. As before, this party can join a coalition as the $i$ th member where $1 \leq i \leq(m+1)$. However, its marginal contribution is one only if it joins as the $i$ th member where $(q-j+1) \leq i \leq q$. Thus, out of all $(m+1)$ possible cases, its marginal contribution is one in $j$ cases. Hence the Shapley value of the large party is:

$$
\varphi_{l}=j /(m+1)
$$

Consider a small player. Since $q>m$, a small player's marginal contribution is one in only one case: if it joins a coalition (that already has the large party in it) as the $(q-j+1)$ th member. Out of the $(m+1)$ ! possible permutations, the number of permutations that satisfy this condition is $(q-j)(m-1)$ !. Hence the Shapley value of each small party is:

$$
\varphi_{s}=(q-j) / m(m+1)
$$


We get the uncertainty for the large party as:

$$
\beta_{l}=\left(1-\varphi_{l}\right) \varphi_{l}^{2}+\varphi_{l}\left(1-\varphi_{l}\right)^{2}
$$

For each small party, the uncertainty is:

$$
\beta_{s}=\left(1-\varphi_{s}\right) \varphi_{s}^{2}+\varphi_{s}\left(1-\varphi_{s}\right)^{2}
$$

Note that for each player, uncertainty (in Equations 4, 5, 8, and 9) has the same relation with Shapley value as that for Equation 1. Therefore, the plot in Figure 1 applies to Equations 4, 5, 8, and 9 as well. Thus, for the voting game with a single large player, each player's uncertainty first increases as its Shapley value increases. A player's uncertainty is at a maximum when its Shapley value is $1 / 2$. As the Shapley value increases further, uncertainty decreases.

\section{Multiple large and multiple small parties}

Consider a parliament in which there are $m$ parties. The set of parties consists of $\mathrm{km}$ large parties and $(1-k) m$ small parties where $0 \leq k \leq 1$. As before, each large party has $j$ seats and each small one has one seat. The total seats in a coalition of size $m$ is $m k j+(1-k) m$. Thus, in a given population of players, the proportion of large players is $k$. Here, the quota $(q)$ satisfies the constraint $(j+1) \leq q \leq(k m j+(1-k) m-1)$. As before, the lower and upper limits for $j$ are 2 and $(q-1)$ respectively. Finally, the value of a coalition is one if it has $q$ or more seats, otherwise it is zero.

A Randomised Method for the Shapley Value. In order to determine a player's Shapley value, we consider a sample from the above defined population of players. Let this sample be a large random coalition of size $X$. Let $\hat{k}$ denote the proportion of large players in this sample. Irrespective of how the population is distributed, the proportion of large players in a sample of size $X$ is distributed approximately normally, with mean $\mu=k$ and variance $\nu=k(1-k) / X$ (see [2] p435), i.e., we have:

$$
\hat{k} \sim \mathcal{N}\left(k, \frac{k(1-k)}{X}\right)
$$

On the basis of Equation 10, we obtain the Shapley value as follows. Consider a large party. The marginal contribution of this party to the random sample is one if the weight of the sample is less than the quota $(q)$ but is greater than or equal to $(q-j)$. Otherwise, its marginal contribution is zero. We know that the mean weight of the sample is $\hat{k} X j+$ $(1-\hat{k}) X$. Let $a$ denote the proportion of large players that is required for the random sample to have mean weight $(q-j)$ (i.e., $a=(q-j-X) /(X(j-1)))$. Also, let $b$ denote the proportion of large players that is required for the random sample to have mean weight $(q-\epsilon)$ (where $\epsilon$ is an infinitesimally small positive number) (i.e., $b=$ $(q-X-\epsilon) /(X(j-1)))$. The expected marginal contribution of a large player to the random sample is the area under the curve defined by the normal distribution of Equation 10 between the limits $a$ and $b$, i.e.,

$$
\Delta_{l}^{X}=\frac{1}{\sqrt{(2 \pi \nu)}} \int_{a}^{b} e^{-\frac{(x-\mu)^{2}}{2 \nu}} d x
$$




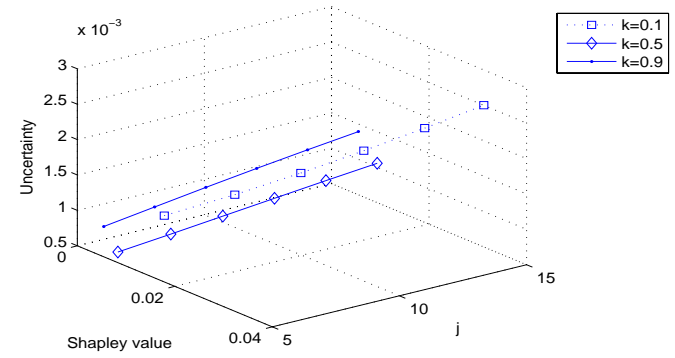

Fig. 2. A large player's Shapley value and uncertainty for a varying weight.

Therefore, a large player's Shapley value is:

$$
\varphi_{l}=\frac{1}{m} \sum_{X=1}^{m} \Delta_{l}^{X}
$$

and its uncertainty is:

$$
\beta_{l}=\frac{1}{m} \sum_{X=1}^{m}\left(\Delta_{l}^{X}-\varphi_{l}\right)^{2}
$$

Consider a small party. The marginal contribution of this party, when added to a sample, is one if the weight of the sample is less than the quota $(q)$ but is greater than or equal to $(q-1)$. Otherwise, its marginal contribution is zero. We know that the mean weight of the sample is $\hat{k} X j+(1-\hat{k}) X$. Let $c$ denote the proportion of large players that is required for the random sample to have mean weight $(q-1)$ (i.e., $c=(q-1-$ $X) /(X(j-1)))$. Also, let $d$ denote the proportion of large players that is required for the random sample to have mean weight $q-\epsilon$ (i.e., $d=(q-X-\epsilon) /(X(j-1)))$. The marginal contribution of a small player is the area under the curve defined by the normal distribution of Equation 10 between the limits $c$ and $d$, i.e.,

$$
\Delta_{s}^{X}=\frac{1}{\sqrt{(2 \pi \nu)}} \int_{c}^{d} e^{-\frac{(x-\mu)^{2}}{2 \nu}} d x
$$

Therefore, for each small player, the Shapley value is:

$$
\varphi_{s}=\frac{1}{m} \sum_{X=1}^{m} \Delta_{s}^{X}
$$

and the uncertainty is:

$$
\beta_{s}=\frac{1}{m} \sum_{X=1}^{m}\left(\Delta_{s}^{X}-\varphi_{s}\right)^{2}
$$




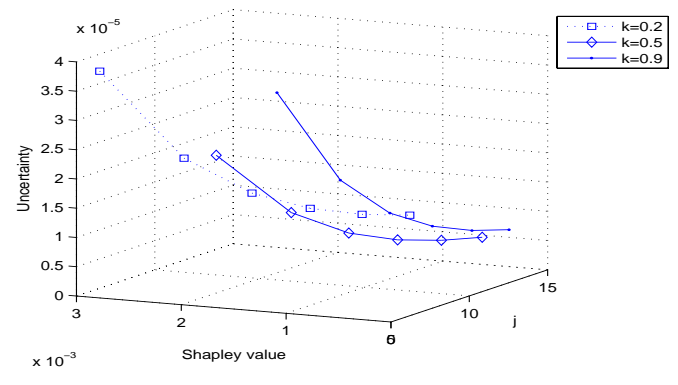

Fig. 3. A small player's Shapley value and uncertainty for a varying weight.

Theorem 1. The time complexity of the above randomised method for determining the Shapley value is polynomial in the number of players. The inaccuracy of this method decreases with $X$ and increases with $\epsilon$.

Proof. The time required to compute the marginal contribution of a player to a coalition of size $i$ (for $1 \leq i \leq m$ ) is independent of the number of players (see Equations 11 and 14). A player can join the coalition as the ith member (for $1 \leq i \leq m$ ). The marginal contribution of a player is determined for each of these $m$ possible cases. Therefore, the time taken to compute the Shapley value is $O(m)$.

The accuracy of the proposed method depends firstly on X. We know from [2], that the inaccuracy in Equation 10 decreases as $X$ increases. Consequently, the inaccuracy of the proposed method decreases with X. The second source of inaccuracy is $\epsilon>0$. It is obvious that the closer $\epsilon$ is to zero, the higher the accuracy. Thus, the inaccuracy of the proposed method increases with $\epsilon$.

We now analyse the relation between the Shapley value and its uncertainty. From the above equations, we know that the Shapley value and its uncertainty depend on three parameters: the number of players $(m)$, the weight associated with each large party $(j)$, and the quota $(q)$ for the game. Thus, we systematically vary these parameters in order to study the relation between a player's Shapley value and its uncertainty. These parameters are varied as follows. We varied $k$ between 0.1 and 0.9 . This is because we want multiple large and multiple small players, and for a large $m$, this range for $k$ gives us that. For each $k$, we varied the parameters $m, j$, and $q$ such that the following two constraints are satisfied:

$C_{1}$ No player can win an election on its own (i.e., $j<q$ ).

$C_{2}$ The maximum number of parties required to win an election is less than the total number of parties (i.e., $q<m k j+(1-k) m)$.

Thus, for each $k$, we determined the Shapley value and its uncertainty for different values of $j$ and $q$ that satisfy constraints $C_{1}$ and $C_{2}$. This entire set of variations was repeated for different values of $m$.

Although a player's Shapley value and its uncertainty vary with $j, k, q$, and $m$, the uncertainty was always found to increase with Shapley value. More specifically, for the 

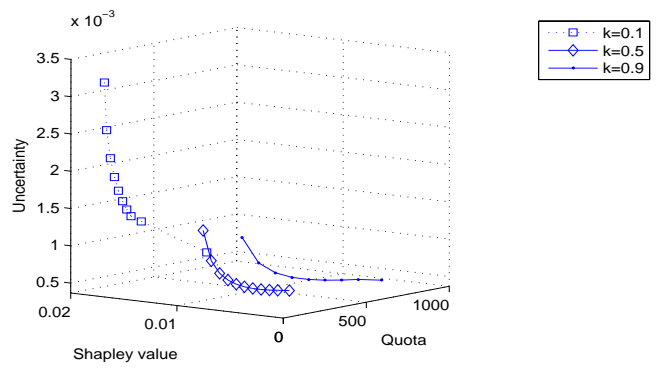

Fig. 4. A large player's Shapley value and uncertainty for a varying quota.

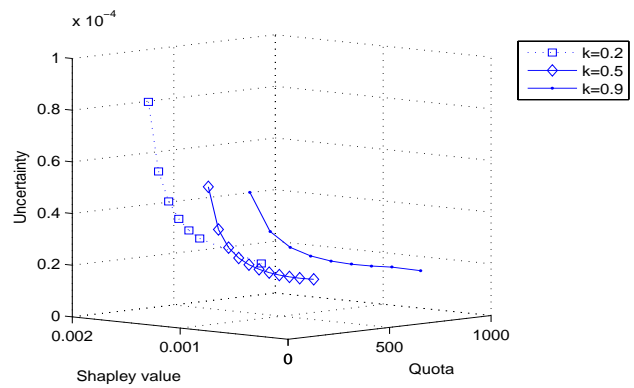

Fig. 5. A small player's Shapley value and uncertainty for a varying quota.

constraints defined above, if the number of players is large (i.e., $m \geq 20$ ) and there are multiple large and multiple small players, we found the following relation. For each player, the uncertainty increases as its Shapley value increases (see Figures 2 to 7).

To begin, consider Figure 2. For $m=200$ and $q=200$, this is a plot of a large player's Shapley value and uncertainty for differing weights (i.e., different values for $j$ ). For each value of $j$, the figure shows the Shapley value and uncertainty for all $k$ between 0.1 and 0.9. Likewise, Figures 3 is a plot for each small player.

For $m=200$ and $j=5$, Figure 4 is a plot for a large player's Shapley value and uncertainty for a varying quota. For each quota (i.e., $q$ ), the figure shows the Shapley value and uncertainty for all $k$ between 0.1 and 0.9 . Figure 5 is a plot for each small player.

Consider Figure 6. For $j=5$ and $q=25$, this is a plot of a large player's Shapley value and uncertainty for a varying number of players. For each $m$, the figure shows the Shapley value and uncertainty for $k$ between 0.1 and 0.9 . Likewise, Figures 7 is a plot for each small player.

Thus, for two player types and variations of $j, k, q$, and $m$ that satisfy constraints $C_{1}$ and $C_{2}$, the uncertainty for each player (large or small) increases as its Shapley value increases. It is worth noting that in all the above figures, the number of players is at 


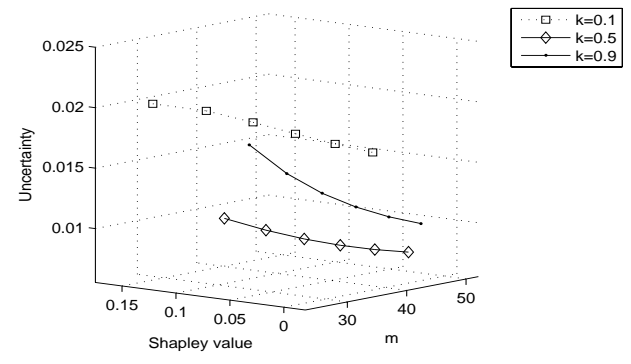

Fig. 6. A large player's Shapley value and uncertainty for a varying number of players.
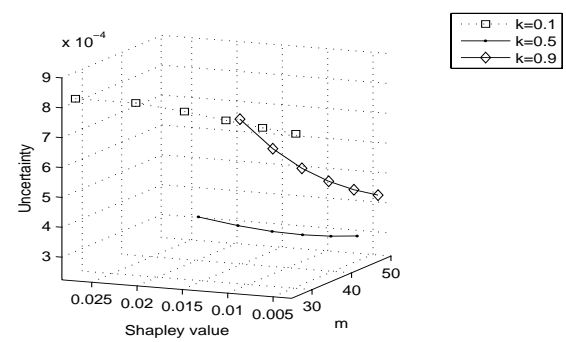

Fig. 7. A small player's Shapley value and uncertainty for a varying number of players.

least 20 , and there is more than one player of each type (i.e., $0.1 \leq k \leq 0.9$ ). For such games, the Shapley value of each player is less than 0.5. Thus, the relation between the Shapley value and its uncertainty is the same as that for the left half of the curve of Figure 1 (i.e., for $\varphi<0.5$ ).

\section{More than two player types}

Consider a voting game with more than two types of players. Let $w_{i}$ denote the weight of player $i$. Thus, for $m$ players and for quota $q$ the game is of the form $\left\langle q ; w_{1}, w_{2}, \ldots, w_{m}\right\rangle$. Consider a player population in which each individual player's weight has a standard normal distribution ${ }^{3}-\mathcal{N}(0,1)$. Since this distribution allows negative weights, we transform this to $\mathcal{N}(4,1)$ in order to get positive weights. We know from Definition 1 ,

\footnotetext{
${ }^{3}$ Note that in Section 6 when we dealt with two player types, there was no restriction on how the population was distributed. But for more than two player types, we assume that the population has a normal distribution. Thus, while the results of Section 6 are valid for two player types with any population distribution, the results of this section are valid for more than two player types that have a normal distribution.
} 


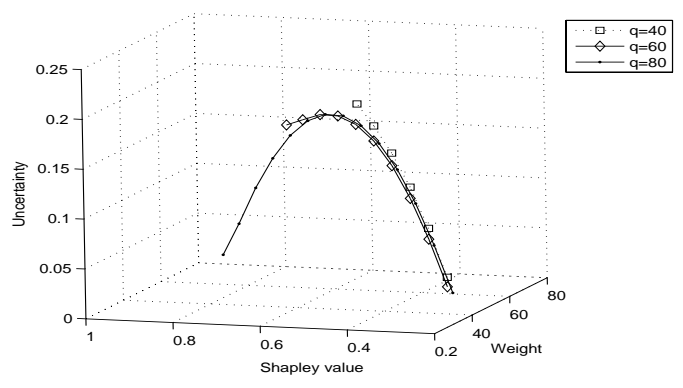

Fig. 8. Shapley value and uncertainty for a game of 20 players and a varying weight.

that the Shapley value for a player is the expectation $(E)$ of its marginal contribution to a coalition that is chosen randomly. Thus, in order to determine the Shapley value for the above population of players (i.e., $\mathcal{N}(4,1)$ ), we use the following rule from Sampling Theory (see [2] p417) that holds good for a normal distribution.

From a normal distribution (with mean $\mu$ and variance $\nu$ ), if a sample of size $m$ is drawn, then the sum of the weights of all $m$ players in the sample has the distribution $\mathcal{N}(m \mu, m \nu)$. Thus, for the distribution $(\mathcal{N}(4,1))$ we defined above, the sum of the weights of the players in a random sample of size $m$ is given by the distribution $\mathcal{N}(4 m, m)$. We use this rule to determine the Shapley value as follows.

A Randomised Method for the Shapley Value. For player $i$ with weight $w_{i}$, let $\varphi_{i}$ denote the Shapley value and $\beta_{i}$ its uncertainty. Let $X$ denote the size of a large random sample drawn from a population in which individual player weights have the distribution $\mathcal{N}(4,1)$. The marginal contribution of player $i$ to this random sample is one, if the total weight of the $X$ players in the sample is greater than or equal to $q-w_{i}$ but less than $q$. Otherwise, its marginal contribution is zero. Thus, the expected marginal contribution of player $i$ (denoted $\Delta_{i}^{X}$ ) to the sample coalition is the area under the curve defined by $\mathcal{N}(4 X, X)$ in the interval $\left[q-w_{i}, q-\epsilon\right]$, i.e.,

$$
\Delta_{i}^{X}=\frac{1}{\sqrt{(} 2 \pi \nu)} \int_{q-w_{i}}^{q-\epsilon} e^{-\frac{(x-4 X)^{2}}{2 X}} d x
$$

and its Shapley value is

$$
\varphi_{i}=\frac{1}{n} \sum_{X=1}^{m} \Delta_{i}^{X}
$$

It is easy to verify that the time complexity of this method is $O(m)$. Also, the two sources of inaccuracy are $X$ and $\epsilon$. As in the case of the randomised method of Section 6 , the inaccuracy decreases with $X$ and increases with $\epsilon$. The uncertainty associated with the Shapley value is:

$$
\beta_{i}=\frac{1}{n} \sum_{X=1}^{m}\left(\varphi_{i}-\Delta_{i}^{X}\right)^{2}
$$




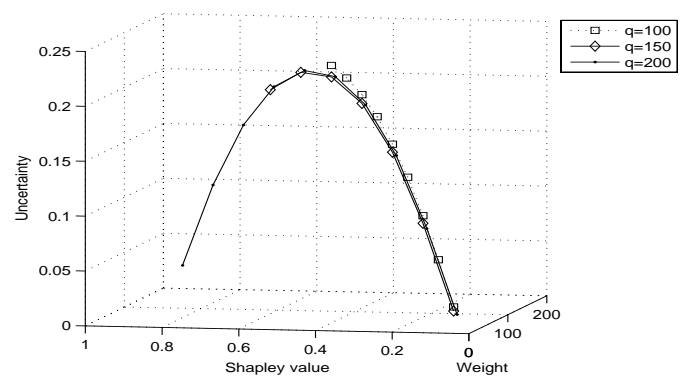

Fig. 9. Shapley value and uncertainty for a game of 50 players and a varying weight.

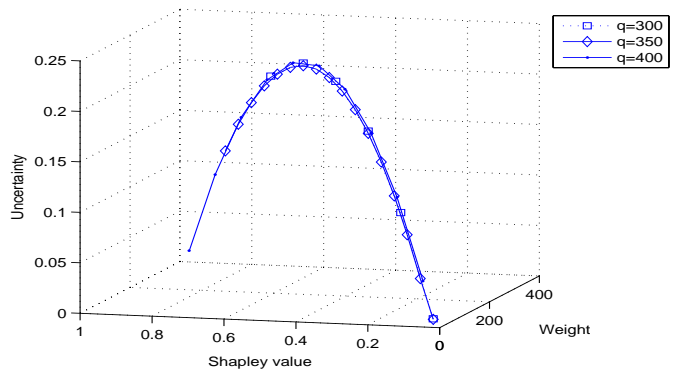

Fig. 10. Shapley value and uncertainty for a game of 100 players and a varying weight.

For the case of more than two player types, we define the following constraints on $q$ and $w_{i}$ (for $\left.1 \leq i \leq m\right)$ :

$C_{3}$ No player can win the game on its own (i.e., $\left(w_{i}<q\right)$ for $\left.1 \leq i \leq m\right)$.

$C_{4}$ The number of players required to win an election is less than $m$ (i.e., the quota is less than $4 m^{2}$ ).

We use the above equations and systematically vary parameters $q, w_{i}$ (for $1 \leq$ $i \leq m$ ), and $m$, such that constraints $C_{3}$ and $C_{4}$ are always satisfied, and determine the relation between the Shapley value and its uncertainty. These results are plotted in Figures 8 to 10. Consider Figure 8. For each quota, an individual player's weight is varied between 1 and $q-1$. As seen in the figure, uncertainty first increases with Shapley value and then decreases. Figure 9 is a plot for $m=50$ and Figure 10 that for $m=100$. In all these figures, a player's uncertainty first increases with its Shapley value and then decreases. Thus, the relation between the Shapley value and its uncertainty is the same as that in Figure 1.

To sum up, our study provides a basis for agents to compare games on the basis of both their Shapley values and the associated uncertainties. We showed that a player's uncertainty first increases with its Shapley value and then decreases. This implies that 
the uncertainty is at its minimum when the value is at its maximum, and that agents do not always have to compromise value in order to reduce uncertainty. This is because, if the Shapley value lies in the range [0.5..1], then an increase in value is associated with a decrease in uncertainty.

\section{Conclusions and future work}

Although the Shapley value provides a unique solution that gives an indication of an agent's power relative to that of others, it also has an element of uncertainty associated with it. Given this, the uncertainty is an additional dimension that an agent should take into account for evaluating its prospects of playing a game. Against this background, this paper has analysed the relation between the Shapley value and its uncertainty for the weighted voting game. Since the problem of determining the Shapley value is \#Pcomplete, we first presented a randomised method with polynomial time complexity. Using this method, we computed the Shapley value and correlated it with its uncer-

tainty. Our study shows that a player's uncertainty first increases with its Shapley value and then decreases. Although our present work provides an analysis for the case where different players have different weights, the distribution of weights was assumed to be normal. In future, we will generalise our results, by extending this analysis to other types of distribution functions. Also, we will carry out the same analysis for other commonly occurring games like the production-economy and the market-economy.

\section{References}

1. X. Deng and C. H. Papadimitriou. On the complexity of cooperative solution concepts. Mathematics of Operations Research, 19(2):257-266, 1994.

2. A. Francis. Advanced Level Statistics. Stanley Thornes Publishers, 1979.

3. J. C. Harsanyi. Rational behavior and bargaining equilibrium in games and social situations. Cambridge University Press, 1977.

4. V. Kargin. Uncertainty of the Shapley value. International Game Theory Review, (To appear), 2004.

5. I. Mann and L. S. Shapley. The bargaining problem. Technical report, The RAND Corporation, Santa Monica, 1960.

6. I. Mann and L. S. Shapley. The bargaining problem. Technical report, The RAND Corporation, Santa Monica, 1962.

7. M. J. Osborne and A. Rubinstein. A Course in Game Theory. The MIT Press, 1994.

8. C. H. Papadimitriou. Computational Complexity. Addison Wesley Longman, 1994.

9. J. S. Rosenschein and G. Zlotkin. Rules of Encounter. MIT Press, 1994.

10. A. E. Roth. The Shapley value as a von Neumann-Morgenstern utility. Econometrica, 45(3):657-664, 1977.

11. A. E. Roth. The expected utility of playing a game. In A. E. Roth, editor, The Shapley value, pages 51-70. University of Cambridge Press, Cambridge, 1988.

12. T. Sandholm and V. Lesser. Coalitions among computationally bounded agents. Artificial Intelligence Journal, 94(1):99-137, 1997.

13. L. S. Shapley. A value for n-person games. In A. E. Roth, editor, The Shapley value, pages 31-40. University of Cambridge Press, Cambridge, 1988.

14. O. Shehory and S. Kraus. Methods for task allocation via agent coalition formation. Artificial Intelligence Journal, 101(2):165-200, 1998. 